\title{
Methotrexate-Loaded Nanostructured Lipid Carrier Gel Alleviates Imiquimod-Induced Psoriasis by Moderating Inflammation: Formulation, Optimization, Characterization, In-Vitro and In-Vivo Studies
}

This article was published in the following Dove Press journal: International Journal of Nanomedicine

\author{
Yogeeta O Agrawal' \\ Umesh B Mahajan (iD) ${ }^{2}$ \\ Hitendra S Mahajan' \\ Shreesh Ojha ${ }^{3}$ \\ 'Department of Pharmaceutics and \\ Quality Assurance, R. C. Patel Institute of \\ Pharmaceutical Education and Research, \\ Shirpur, Maharashtra 425405, India; \\ ${ }^{2}$ Department of Pharmacology, \\ R. C. Patel Institute of Pharmaceutical \\ Education and Research, Shirpur, \\ Maharashtra 425405, India; ${ }^{3}$ Department \\ of Pharmacology and Therapeutics, \\ College of Medicine and Health Sciences, \\ United Arab Emirates University, Al Ain, \\ Abu Dhabi, United Arab Emirates
}

Correspondence: Yogeeta O Agrawal R. C. Patel Institute of Pharmaceutical Education and Research, Shirpur, District-Dhule, Maharashtra 425405 Tel +02563255189 (Office)

Email goyalyogita@rediffmail.com
Introduction: Methotrexate exhibits poor cutaneous bioavailability and systemic side effects on topical administration, so there is an unmet need for a novel carrier and its optimized therapy. Methotrexate-loaded nanostructured lipid carriers (MTXNLCs) were formulated and characterized to determine in vitro drug release and evaluate the role of MTXNLC gel in the topical treatment of psoriasis.

Methods: A solvent diffusion technique was employed to prepare MTXNLCs, which was optimized using $3^{2}$ full factorial designs. The mean diameter and surface morphology of MTXNLCs was evaluated. The crystallinity of lyophilized MTXNLCs was characterized by differential scanning calorimetry (DSC) and powder X-ray diffraction (XRD). MTXNLCs were integrated in 1\% w/w Carbopol $934 \mathrm{P}$ gel base, and in vitro skin deposition studies in human cadaver skin (HCS) were carried out.

Results: The optimized MTXNLCs were rod-shaped, with an average particle size of $253 \pm$ $8.65 \mathrm{~nm}$, a zeta potential of $-26.4 \pm 0.86 \mathrm{mV}$, and EE of $54.00 \pm 1.49 \%$. DSC and XRD data confirmed the formation of NLCs. Significantly higher deposition of MTX was found in HCS from MTXNLC gel $(71.52 \pm 1.13 \%)$ as compared to MTX plain gel $(38.48 \pm 0.96 \%)$. In vivo studies demonstrated significant improvement in therapeutic response and reduction in local side effects with MTXNLCs-loaded gel in the topical treatment of psoriasis. Anti-psoriatic efficacy of MTXNLCs $100 \mathrm{ug} / \mathrm{cm}^{2}$ compared with plain MTX gel was evaluated using imiquimod (IMQ)-induced psoriasis in BALB/c mice. The topical application of MTXNLCs to the mouse ear resulted in a significant reduction of psoriatic area and severity index, oxidative stress, inflammatory cytokines like TNF- $\alpha$, IL-1 $\beta$, and IL-6 and IMQ-induced histopathological alterations in mouse ear samples.

Conclusion: Developed formulation of MTXNLC gel demonstrated better anti-psoriatic activity and also displayed prolonged and sustained release effect, which shows that it can be a promising alternative to existing MTX formulation for the treatment of psoriasis.

Keywords: methotrexate, nanostructured lipid carriers, Capmul MCM, Compritol 888, psoriasis, IMQ

\section{Introduction}

Psoriasis is considered as chronic inflammatory skin disease and is clinically characterized by erythematous, sharply demarcated papules and rounded plaques 
covered by silvery micaceous scale, epidermal hyperproliferation overlying immune-mediated dermal inflammation, leading to profound adverse effects on patients' physical, social, and mental well-being (affecting millions of people worldwide). ${ }^{1}$ Management of psoriasis can be done based on the symptoms and major factors affecting the disease like occurrence, causative factors, the behavior of the disease in different individuals, aggravating factors, the efficacy of the available drugs, cost of different therapies on a long-term basis, etc. ${ }^{2}$ Topical therapies currently available for the treatment of psoriasis include tar, methoxsalen (psoralen) with ultraviolet $\mathrm{A}$ radiation (PUVA), calcipotriene, corticosteroids, calcineurin inhibitors, tacrolimus, pimecrolimus, tazarotene, vitamin D analogs, and salicylic acid. Moreover, systemic treatment of psoriasis includes drugs like acitretin, cyclosporine, methotrexate, infliximab, ixekizumab, secukinumab, anthralin phototherapy with ultraviolet B radiation (UVB), and apremilast, also the biologics such as TNF-alpha blockers (etanercept, infliximab, adalimumab), IL-17 blockers (secukinumab, ixekizumab), and IL-23 blockers (guselkumab, risankizumab) $)^{2,3}$ Nevertheless, none of them are entirely secure and effective to treat the disease without compromising patient compliance. ${ }^{2}$ Topical treatments suppress the body's immune system (eg, topical steroids) when used for a prolonged period of time, whereas systemic drugs impair liver and kidney functioning and decrease red blood cell (RBC), white blood cell (WBC) and platelet counts.

Nanostructured lipid carriers (NLCs), a new generation of drug carrier, consist of a lipid matrix with a special nanostructure. They are the second generation of solid lipid nanoparticles (SLN). Unlike SLNs (a highly ordered structure due to involvement of solid lipids or blends of solid lipids), NLCs present a lipid matrix with great imperfections in the crystal lattice, having enough space to accommodate drug molecules, leading to improved drug loading capacity, preventing its leakage, and giving more flexibility for modulation of drug release. ${ }^{4-7}$

This may be due to the incorporation of liquid lipids into solid lipids, which leads to massive disturbances in their crystal order. Due to their biocompatibility and capability of incorporating both hydrophilic and lipophilic drugs, NLCs have been widely investigated in topical delivery systems and cosmetic products. Their small particle size ensures close contact with the stratum corneum and the lipid provides selective drug delivery to skin layers. ${ }^{8-11}$ NLCs possess a solid matrix, which has the potential to modulate the drug release over a prolonged period with a reduced rate of systemic absorption. ${ }^{11}$ Another benefit of NLCs for topical delivery of active compounds is the short time required to market these products. $^{12-14}$

Methotrexate (MTX) is a folic acid antagonist with antineoplastic activity. It is effective for the treatment of psoriasis when administered by the oral or parenteral route over long periods of time. However, the systemic use of MTX is associated with side effects such as hepatotoxicity. ${ }^{13}$ Topical administration of MTX has shown promise for improvement of therapy with reduced side effects, which is evidenced from many clinical studies ${ }^{15}$. However, major drawbacks associated with the drug for topical use include its poor penetration across the skin due to its hydro solubility and high molecular weight $(454.56 \mathrm{~g} / \mathrm{mol}) .{ }^{16}$ Therefore, there is a need to develop new delivery systems to solve this problem and improve the local bioavailability of methotrexate. One of the possibilities includes the development of NLCs to increase the penetration of MTX across the skin. Hence, the present work aimed to explore the potential benefits of NLCs in improving the topical delivery of MTX for the treatment of psoriasis.

Imiquimod is a synthetic nucleoside analog of the imidazoquinoline family. ${ }^{17}$ It was discovered by compound screens for anti-herpes virus activity. ${ }^{18}$ Topical application of the compound is currently approved for the treatment of genital and anal wart.

Topical application of IMQ on the skin and ears of mice leads to psoriasis-like dermatitis with many hallmarks of human psoriasis including skin thickening, hyperkeratosis, acanthosis, scaling, and erythema.

\section{Materials and Methods Materials}

Methotrexate was obtained as a gift from Zydus Cadila, Ahmadabad, India. Compritol 888 was obtained as a gift sample from Gattefosse, Saint-Priest, France. Capmul MCM C8 was received as a gift sample from Abitex limited, India. A dialysis bag (molecular weight cutoff of $14 \mathrm{kDa}$ ) was purchased from Hi-media Pvt. Ltd, Mumbai, India. Trehalose dehydrate was purchased from Sigma Chemicals Co., USA. Carbopol 934P (Noveon, USP) was obtained from BF Goodrich (Cleveland OH, USA), propylene glycol, and glycerol were purchased from Qualigens, Mumbai, India. HCS (forearm region of the dead bodies) was obtained from All India Institute of Medical Sciences Hospital, Delhi, India (no ethical 
approval required for using HCS). All other chemicals used were of analytical grade or spectroscopic grade.

\section{Preparation of MTX Loaded NLC}

Drug-loaded NLCs were prepared by the solvent diffusion method in an aqueous system. ${ }^{19}$ Briefly, Capmul MCM and MTX (5\%) were added to the melted Compritol 888, $4 \mathrm{~mL}$ of acidified isopropyl alcohol (IPA) was added and the resultant organic phase was heated to $65-70^{\circ} \mathrm{C}$ in a water bath till the entire lipid was dissolved in solvent. This phase was then added dropwise into $25 \mathrm{~mL}$ of distilled water under mechanical agitation using a high-speed homogenizer, Eurostar (IKA Labortechnik, Staufen, Germany) at $1000 \mathrm{rpm}$ for $10 \mathrm{~min}$. The obtained preemulsion was transferred to a round bottom flask and subjected to Rota evaporation at $70^{\circ} \mathrm{C}$ to remove the residual solvent under reduced pressure. After complete removal of organic solvent, the dispersion was cooled under stirring at room temperature, yielding drug-loaded NLCs. After the $\mathrm{pH}$ value of the obtained NLC suspensions were adjusted to 1.20 by the addition of 0.1 $\mathrm{M}$ hydrochloric acid, the nanoparticles were aggregated and centrifuged at 25,000 rpm for $30 \mathrm{~min}$ (3K30, Sigma, Germany). The NLC pellet was re-dispersed in distilled water and the resultant dispersion was kept in a deepfreezer (Sanyo Ultra Low Temperature Freezer MDF192, Moriguchi, Japan) at $-75^{\circ} \mathrm{C}$ for $5 \mathrm{~h}$. The sample was then subjected to freeze-drying (Freezone $2.5 \mathrm{~L}$, Labconco, Kansas City, MO, USA) using trehalose as a cryoprotectant in the weight ratio of 1:1 with the total solid content (samples). A similar procedure was followed for the preparation of blank (without using the drug) NLC dispersion.

\section{Experimental Design}

Optimization of MTX loaded NLCs was carried out by $3^{2}$ factorial design to determine the effect of the two independent variables, amount of Compritol 888 (solid lipid), and amount of Capmul MCM (liquid lipid) on mean particle size (MPS) and entrapment efficiency (EE) (response variables). Each factor was tested at three levels designated as $-1,0$, and +1 . The values of the factors were transformed to allow easy calculation of coefficient in the polynomial equation. Interactive multiple regression analysis and F statistics were utilized to evaluate the response and the regression equation for the two responses were calculated using the following formulae:

$$
\begin{aligned}
\text { Response : } & \text { Y1 (MPS) } \\
= & \mathrm{b}_{0}+\mathrm{b}_{1} \mathrm{X}_{1}+\mathrm{b}_{2} \mathrm{X}_{2}+\mathrm{b}_{1}{ }^{2} \mathrm{X}_{11}+\mathrm{b}_{2}^{2} \mathrm{X}_{22}+\mathrm{b}_{12} \mathrm{X}_{1} \mathrm{X}_{2}
\end{aligned}
$$

$$
\begin{aligned}
& \text { Response : } \mathrm{Y} 2(\mathbf{E E})=\mathrm{b}_{0}+\mathrm{b}_{1} \mathrm{X}_{1}+\mathrm{b}_{2} \mathrm{X}_{2}+\mathrm{b}_{1}^{2} \mathrm{X}_{11} \\
& +b_{2}{ }^{2} X_{22}+b_{12} X_{1} X_{2}
\end{aligned}
$$

Where, Y1 and Y2 are dependent variables viz. MPS and $\mathrm{EE}$, respectively, indicating the quantitative effect of the formulation components, while $b_{0}$ is the intercept and $b_{1}$, $b_{2}$ represent the regression coefficients for the secondorder polynomial equations. The amount of solid lipid $\left(\mathrm{X}_{1}\right)$ and liquid lipid $\left(\mathrm{X}_{2}\right)$ represents the level of independent variables. $\mathrm{X}_{1}^{2}\left(\mathrm{X}_{11}\right), \mathrm{X}_{2}^{2}\left(\mathrm{X}_{22}\right)$ and $\mathrm{X}_{1} \mathrm{X}_{2}$ stands for the quadratic effects of the variables. $\mathrm{X}_{1} \mathrm{X}_{2}$ indicates the interactions between two factors. Multiple regression was applied using Microsoft Excel in order to deduce the factors having a significant effect on the responses. The variables having $p$-value $<0.05$ in the model were considered to have a significant effect on the formulations. ${ }^{20}$

\section{Contour Plots and Surface Response Curves}

Contour plots and surface response curves represent the values of the response and help in explaining the relationship between independent and dependent variables. The full models were used to plot both the contour plots and surface response curves using Microsoft Excel and STATISTICA ${ }^{\circledR}$ students' version 1998 at the values of $X_{1}$ and $X_{2}$ between -1 and +1 at predetermined values of particle size and EE. ${ }^{21}$ Full model equations for MPS and EE were developed as per equations 1 and 2, respectively.

\section{Characterization of MTX Loaded NLCs MPS, Poly-Dispersity Index, and Zeta Potential}

The MPS, poly-dispersity index (PI) and zeta potential of blank and MTXNLCs were determined by laser light diffractometry using Zetasizer nanoseries, SM 2000K (Malvern Instruments Inc., Malvern, UK). Samples were prepared by dispersing MTXNLCs in a sufficient amount of water ( $\mathrm{pH} 7)$ to achieve obscuration between $10 \%$ and $20 \%$.

\section{Entrapment Efficiency (EE)}

The free drug (unentrapped) in the NLC dispersion was sedimented by controlled centrifugation at $2000 \mathrm{rpm}, 4^{\circ} \mathrm{C}$ for 30 min (Sigma centrifuge, 3K30, Sigma, Germany) and the NLC dispersion was decanted without disturbing the sedimented drug pellet. NLC dispersion was used for the estimation of the entrapped drug, whereas the amount of unentrapped drug was determined from the MTX pellet. 
A fixed volume of NLC dispersion and MTX pellet was dissolved in acidified methanol: chloroform (MeOH: $\mathrm{CHCl}_{3}$ in a ratio of 1:2) and phosphate buffer $\mathrm{pH} 7.4$ and analyzed for entrapped and unentrapped drug, respectively, using UV spectroscopy at $303 \mathrm{~nm}$ (Shimadzu 1601, Kyoto, Japan). EE was calculated using equation 3 .

$$
\begin{aligned}
\mathrm{EE} & =\frac{\text { Entrapped Drug }}{\text { Entrapped Drug }+ \text { Free Drug }} \times 100 \% \mathrm{EE} \\
& =\frac{\text { Actual amount of drug entrapped in NLCs }}{\text { Theorotical amount of drug entrapped in NLCs }} \times 100
\end{aligned}
$$

\section{Surface Morphology by Cryo-Transmission Electron Microscopy}

The morphological observation of MTX loaded NLCs was performed to analyze the size, size distribution, and shape of the NLC particles by Cryo-Transmission Electron Microscopy (TEM) (CEM902a, Zeiss, D-Oberkochen, Philips CM120, NLEindhoven) after sufficient dilution. ${ }^{22}$

\section{Differential Scanning Calorimetry}

DSC studies of pure MTX, Compritol 888, physical mixture of MTX and Compritol 888 (at a ratio equivalent to that in the formulation) and the lyophilized MTXNLC powder were carried out. Accurately weighed samples were carefully placed in DSC boats and heating curves were recorded in the temperature range of $25-250^{\circ} \mathrm{C}$ at a heating rate of $10^{\circ} \mathrm{C} / \mathrm{min}$ under an inert atmosphere $\left(\mathrm{N}_{2}\right.$ $8 \mathrm{~mL} / \mathrm{min}$ ). The study was carried out using a differential scanning calorimeter (DSC, Mettler Toledo e822, Japan).

\section{X-Ray Diffraction Study}

An XRD study was carried out to judge the crystalline or amorphous nature of the MTX loaded NLCs. X-ray powder diffraction studies of pure MTX, Compritol 888, physical mixture of MTX and Compritol 888 (at a ratio equivalent to that in the formulation) and lyophilized MTXNLCs were done using Bruker AXS D8 Advance $\mathrm{X}$-ray diffractometer from $2^{\circ}$ to $80^{\circ}$ on $2 \theta$ scales.

\section{In vitro Release Study}

The in vitro drug release profile of the MTXNLCs was determined by dispersing $20 \mathrm{mg}$ of the lyophilized MTXNLCs in $25 \mathrm{~mL}$ of phosphate buffer saline (PBS, $\mathrm{pH}$ 7.4), placing it into the dialysis bag (cutoff $14 \mathrm{kDa}$ ) and stirring on a magnetic stirrer at $100 \mathrm{rpm}$ at $37 \pm 1^{\circ} \mathrm{C}$. One $\mathrm{mL}$ of the dispersion was withdrawn from the medium and replaced with the same amount of medium at a definite time interval. It was filtered using a $0.4 \mu \mathrm{m}$ filter (EMD Millipore, Billerica, MA, USA) and drug release was analyzed by UV-visible spectroscopy at $303 \mathrm{~nm}$. Similarly, the in vitro drug release profile of the MTX suspension was determined by suspending $2.5 \mathrm{mg}$ of MTX in $25 \mathrm{~mL}$ of PBS (pH 7.4). The experiments were run in triplicate and the average results are reported.

\section{Preparation of MTX Loaded NLCs Gel}

Carbopol $934 \mathrm{P}$ was used to prepare MTXNLCs gel. In brief, Carbopol $934 \mathrm{P}(1 \%)$ was dispersed in distilled water and allowed to hydrate for 4 to $5 \mathrm{~h}$. Propylene glycol (10\%) and glycerol (30\%) were added subsequently to the aqueous dispersion, and $0.5 \mathrm{~mL}$ of triethanolamine was added under gentle stirring to avoid the inclusion of air. ${ }^{23}$ Lyophilized MTXNLC powder equivalent to $600 \mathrm{mg}$ of the drug was incorporated into the gel under gentle stirring. The dispersion was neutralized with $1 \% \mathrm{w} / \mathrm{v} \mathrm{NaOH}$ to $\mathrm{pH} 6.0$, and water (58.4\%) was added to make up the final weight of gel up to $100 \%$.

\section{In vitro Skin Deposition Study}

HCS was washed thoroughly with hot distilled water to remove subcutaneous fat. Full thickness HCS membrane was prepared by shaving the skin, punching out a disc of approximately $2.5 \mathrm{~cm}^{2}$ areas, and slicing it to $500 \mathrm{~mm}$ thickness using a Davis Dermatome-7 (Anthony Products, Indianapolis, IN, USA). These slices were hydrated with PBS for $24 \mathrm{~h}$ at room temperature prior to use. ${ }^{24}$ Thereafter, $0.1 \mathrm{~g}$ of gel was weighed, spread over the skin, and allowed to stand for $36 \mathrm{~h}$ at room temperature. The amount of drug remaining on the skin surface was determined by washing the skin surface thrice with PBS and measuring the absorbance of the drug in PBS at $303 \mathrm{~nm}$. The residual washing solvent on the skin was carefully wiped off with a cotton swab, the skin was digested overnight in PBS containing 3\% $\mathrm{w} / \mathrm{v}$ sodium lauryl sulfate (SLS) at $40^{\circ} \mathrm{C}$ to allow the escape of drug from the skin and the drug escaped from the skin was quantified by a UV-visible spectrophotometer at $303 \mathrm{~nm}$ to determine drug deposited in the skin.

\section{In vivo Study}

\section{Animals (Ethics Statement)}

$\mathrm{Balb} / \mathrm{c}$ mice (7-8 weeks old) were procured from the laboratory animal facility of our institute. They were housed in standard temperature $\left(22^{\circ} \mathrm{C}\right)$ /relative humidity $(65 \%)$ conditions and the environment with a $12 \mathrm{~h}$ photocycle. All animals were provided a standard pellet diet and water ad libitum. The animals were maintained in conformity with the regulations laid down by the Committee for the Purpose 
of Control and Supervision of the Experiments on Animals (CPCSEA) constituted under the Prevention of Cruelty to Animals Act, 1960, Ministry of Environment and Forests, Government of India. The experimental protocols were approved by the IAEC of R. C. Patel Institute of Pharmaceutical Education and Research, Shirpur, DistDhule, Maharashtra, India.

\section{Imiquimod-Induced Psoriasis in Mice}

Balb/c mice (7-8 weeks old) were used for in vivo evaluation of the anti-psoriatic activity of methotrexate-loaded nanostructured lipid carriers (MTXNLCs). ${ }^{25,26}$ Psoriasis was induced in mice by topical application of IMQ on ear. Daily, $3.5 \mathrm{mg}$ of IMQ mixed with soft paraffin was applied on the right ear of each mouse from all the groups except the vehicle control group (receives soft paraffin only). This application continued for 6 days. The left ear of each mouse and both ears of the vehicle control group were applied with soft paraffin as a vehicle for 6 days.

\section{Treatment Groups}

Mice were randomly divided into four groups $(n=6)$.

Group I: Vehicle control; applied with soft paraffin on both ears.

Group II: IMQ treated; right ear applied with $3.5 \mathrm{mg}$ of IMQ/per day and left ear with soft paraffin.

Groups III: Methotrexate carbopol gel (MTXC) treated; right ear applied with $3.5 \mathrm{mg} \mathrm{IMQ} /$ per day and left ear with soft paraffin. Topically, MTXC $\left(100 \mu \mathrm{g} / \mathrm{cm}^{2}\right)$ was applied after an hour of IMQ application for 6 days.

Groups IV: Methotrexate-loaded nanostructured lipid carriers (MTXNLCs) treated; right ear applied with $3.5 \mathrm{mg} \mathrm{IMQ} / \mathrm{per}$ day and left ear with soft paraffin. Topically, MTXNLCs $\left(100 \mu \mathrm{g} / \mathrm{cm}^{2}\right)$ was applied after an hour of IMQ application for 6 days.

\section{Pharmacological Evaluation Measurement of Ear Thickness}

The ear thickness was measured using a Vernier caliper (Digimatic Caliper, Mitutoyo Products) at 0, 2, 4, 6 days. The increase in ear thickness was used to indicate the extent of inflammation. ${ }^{27}$

\section{PASI Score}

PASI scoring of the mice was done to examine the severity of psoriasis in the IMQ-induced mice by evaluating the degree of erythema, swelling, and scaling on the affected skin surface, an objective scoring system was developed based on the clinical Psoriasis Area and Severity Index (PASI). PASI score was measured on a scale of 0 to 4 , from none to maximum. 0-none; 1-slight; 2-moderate; 3-marked; 4-very marked. The cumulative score (0-12) of erythema, swelling, and scaling indicated the severity of inflammation. ${ }^{28}$

\section{Ear Weight}

At the end of the study, mice were sacrificed, and using a biopsy punch $(4 \mathrm{~mm})$ ear tissue was collected from both the ears. The difference in IMQ-treated ear and control ear was calculated in percentage increase in weight.

\section{Weight of Lymph Node, Spleen, and Thymus}

Auricle lymph node, spleen, and thymus were isolated after $24 \mathrm{~h}$ of the final application of IMQ. The lymph node of both ears was collected also spleen and thymus isolated. The weight of these organs was taken immediately after the isolation.

\section{Tissue Homogenization}

After completion of treatments, animals were bled under anesthesia and then sacrificed. Ear samples were collected and kept in liquid nitrogen and they were homogenized in phosphate buffer saline ( $\mathrm{pH} 7.4)$ and stored at $-20^{\circ} \mathrm{C}$ for further assays.

\section{Estimation of Oxidative Stress Estimation of GSH}

The GSH content in ear homogenates was determined by treating the homogenates with the DTNB method..$^{29}$ Briefly, $20 \mu \mathrm{L}$ of tissue homogenate was treated with $180 \mu \mathrm{L}$ of $1 \mathrm{mM}$ DTNB solution at room temperature. The optical density of the resulting yellow color was measured at $412 \mathrm{~nm}$ using a microplate spectrophotometer and final concentration was determined by using standard calibration curve (Powerwave XS, BioTek Instruments Inc., Winooski, VT, USA).

\section{Determination of the Catalase Activity}

As reported, ${ }^{30}$ the ear homogenate $(20 \mu \mathrm{L})$ was added to $1 \mathrm{~mL}$ of $10 \mathrm{mM} \mathrm{H}_{2} \mathrm{O}_{2}$ solution in the quartz cuvette. The reduction in optical density of this mixture was monitored using a microplate spectrophotometer in UV mode at 240 $\mathrm{nm}$. Rate of decrease in the optical density across $3 \mathrm{~min}$ from the addition of the liver homogenate was taken as an indicator of the catalase activity present in the homogenate 
and the final concentration was determined by using a standard calibration curve.

\section{Estimation of SOD Activity}

The ear homogenate $(20 \mu \mathrm{L})$ was added to a mixture of 20 $\mu \mathrm{L}$ of $500 \mathrm{mM}$ of $\mathrm{Na}_{2} \mathrm{CO}_{3}, 2 \mathrm{~mL}$ of $0.3 \%$ Triton $\mathrm{X}-100,20$ $\mu \mathrm{L}$ of $1.0 \mathrm{mM}$ of EDTA, $5 \mathrm{~mL}$ of $10 \mathrm{mM}$ of hydroxylamine and $178 \mathrm{~mL}$ of distilled water. To this mixture, $20 \mu \mathrm{L}$ of $240 \mu \mathrm{M}$ of NBT was added. The optical density of this mixture was measured at $560 \mathrm{~nm}$ in kinetic mode for 3 $\min$ at $1 \mathrm{~min}$ intervals. The rate increase in the optical density was determined as an indicator of the SOD activity. The final concentration of SOD was determined by using a standard calibration curve.

\section{The Extent of Lipid Peroxidation}

The ear homogenate samples were treated with $3.0 \mathrm{~mL}$ of $1 \%$ phosphoric acid solution and $1.0 \mathrm{~mL}$ of an aqueous solution of $0.6 \%$ thiobarbituric acid. The reaction mixtures were heated at $80^{\circ} \mathrm{C}$ for $45 \mathrm{~min}$, cooled in an ice bath, and extracted with $4.0 \mathrm{~mL}$ of $\mathrm{N}$-butanol. The n-butanol layer was separated and the absorbance of the pink complex formed was estimated at $532 \mathrm{~nm}$ as an indicator of the extent of lipid peroxidation and the final concentration was determined by using a standard calibration curve.

\section{Determination of Pro-Inflammatory Cytokines}

The concentrations of TNF- $\alpha$, IL- 6 , and IL- $1 \beta$ in ear homogenates were determined by sandwich ELISA according to the manufacturer's protocols using ELISA kits. A $100 \mu \mathrm{g}$ protein sample was used for the determination of cytokine level and optical density was taken at 570 $\mathrm{nm}$ by using a microplate reader. Concentrations of cytokine were calculated in $\mathrm{pg} / \mathrm{mg}$ of protein.

\section{Histopathological Examination}

At the end of the experiment, tissue was collected and stored in formalin up to further process. Tissues were processed into paraffin tissue blocks using routine methods, sectioned, or serially sectioned to obtain consecutive levels. The sections were stained with hematoxylin and eosin (H\&E). The grading of various histological parameters was done with reference to the identification of the characteristics of IMQ-induced psoriasis, eg, minimal, mild, moderate, marked, and severe infiltrates observed within the dermis. At least 5-6 fields were analyzed for each slide for proper interpretation. ${ }^{31}$

\section{Statistics}

Data were analyzed mean \pm standard error mean by oneway ANOVA followed by Bonferroni's multiple comparison, repeated measure ANOVA followed by Friedman nonparametric test.

\section{Results and Discussion}

\section{Factorial Design}

Nine batches of MTXNLCs were prepared by the solvent diffusion method utilizing $3^{2}$ statistical factorial design experiments. Table 1 provides the real values and the transformed values for different batches of MTXNLCs along with their MPS and EE. The results of the regression output and response of the full model of MTXNLCs are presented in Table 2. The mean particle size (dependent variable) of MTXNLCs obtained at various levels of the two independent variables $\left(\mathrm{X}_{1}\right.$ and $\left.\mathrm{X}_{2}\right)$ was subjected to multiple regression analysis which resulted in a full model second-order polynomial equation. The MPS values showed a wide variation ranging from $175 \pm 20.1$ to 269 $\pm 10.28 \mathrm{~nm}$, while EE values varied from $34 \pm 1.44 \%$ to 54 $\pm 1.49 \%$. Highest EE was achieved $(54 \pm 1.49 \%)$ at a high level $(+1)$ of $\mathrm{X}_{1}(100 \mathrm{mg})$ and medium level $(0)$ of $\mathrm{X}_{2}$ $(30 \mathrm{mg}$ ) in batch FA8. The following equations (4 and 5) are the quantitative effect of the formulation components on independent variables $X_{1}$ and $X_{2}$. These equations were derived by the best-fit method to describe the relationship between the particle sizes (Y1), the concentration of solid lipid (X1), and the amount of liquid lipid (X2). Similarly, it also describes the relationship between the entrapment efficiency (Y2) and the variables X1 and X2.

$$
\begin{aligned}
\mathrm{Y}_{1}(\mathrm{MPS})= & 233+18 \mathrm{X}_{1}-28.3333 \mathrm{X}_{2}+2 \mathrm{X}_{11} \\
& -7 \mathrm{X}_{22}-4.75 \mathrm{X}_{1} \mathrm{X}_{2} \\
\mathrm{Y}_{2}(\mathrm{EE})= & 52+4.5 \mathrm{X}_{1}-1.5 \mathrm{X}_{2}-2.5 \mathrm{X}_{11}-7.5 \mathrm{X}_{22} \\
+ & 1.75 \mathrm{X}_{1} \mathrm{X}_{2}
\end{aligned}
$$

The significance of each coefficient of equations 4 and 5 was determined on the basis of $p$-values as listed in Table 2. The smaller the $p$-value, the more significant is the corresponding coefficient, implying that the amount of solid lipid and liquid lipid is significant. From Table 2, it is clear that the main effects of the independent variables $\left(\mathrm{X}_{1}\right.$ and $\left.\mathrm{X}_{2}\right)$ were significant factors for prediction of both MPS and EE as their $p$-values are below $0.05(p<0.05)$, whereas the interaction term $\mathrm{X}_{1} \mathrm{X}_{2}$ had little predictive value as their $p$-values were found to be above 0.05 
Table I Formulation of Methotrexate-Loaded NLCs by $3^{2}$ Factorial Design: Factors, Their Levels and Transformed Values, Response: MPS and EE

\begin{tabular}{|c|c|c|c|c|c|c|c|c|c|}
\hline Batch No. & $\begin{array}{l}\text { Compritol } 888 \\
(\mathrm{mg})\end{array}$ & $\begin{array}{l}\text { Capmul MCM } \\
\text { (mg) }\end{array}$ & $\mathbf{X I}$ & $\times 2$ & XII & $\times 22$ & $\times 1 \times 2$ & $\begin{array}{l}\text { MPS } \pm \text { SD* } \\
(\mathrm{nm})\end{array}$ & $\begin{array}{l}\text { EE } \pm \text { SD* } \\
(\%)\end{array}$ \\
\hline Al & 60 & 20 & -1 & -1 & 1 & I & I & $240 \pm 15.9$ & $42 \pm 0.86$ \\
\hline $\mathrm{A} 2$ & 60 & 30 & -1 & 0 & 1 & 0 & 0 & $222 \pm 15.02$ & $44 \pm 1.25$ \\
\hline $\mathrm{A} 3$ & 60 & 40 & -1 & I & I & I & -1 & $175 \pm 20.1$ & $34 \pm 1.44$ \\
\hline A4 & 80 & 20 & 0 & -1 & 0 & I & 0 & $258 \pm 12.2$ & $44 \pm 1.62$ \\
\hline A5 & 80 & 30 & 0 & 0 & 0 & 0 & 0 & $228 \pm 14.5$ & $53 \pm 1.04$ \\
\hline A6 & 80 & 40 & 0 & I & 0 & I & 0 & $199 \pm 20.44$ & $44 \pm 1.16$ \\
\hline A7 & 100 & 20 & 1 & -1 & 1 & I & -1 & $269 \pm 10.28$ & $47 \pm 0.66$ \\
\hline A8 & 100 & 30 & 1 & 0 & I & 0 & 0 & $253 \pm 8.65$ & $54 \pm 1.49$ \\
\hline A9 & 100 & 40 & 1 & I & I & I & I & $223 \pm 12.32$ & $46 \pm 1.66$ \\
\hline
\end{tabular}

Note: *Data are represented as mean \pm SD $(n=3)$.

Table 2 Response of Full Model for Methotrexate-LoadedNLCs

\begin{tabular}{|l|l|l|l|l|}
\hline \multirow{2}{*}{ Response } & \multicolumn{2}{l|}{$\begin{array}{l}\text { Mean Particle Size } \\
\text { (MPS) }\end{array}$} & \multicolumn{2}{l|}{$\begin{array}{l}\text { \%Entrapment } \\
\text { Efficiency (\%EE) }\end{array}$} \\
\cline { 2 - 5 } & $\begin{array}{l}\text { X } \\
\text { Coeff. }\end{array}$ & p-value & $\begin{array}{l}\text { X } \\
\text { Coeff. }\end{array}$ & P-value \\
\hline X1 & 18 & 0.161377 & 4.5 & 0.008793 \\
X2 & -28.333 & 0.003319 & -1.5 & 0.134292 \\
X12 & 2 & 0.000876 & -2.5 & 0.144688 \\
X22 & -7 & 0.620082 & -7.5 & 0.009798 \\
XIX2 & 4.75 & 0.149443 & 1.75 & 0.147502 \\
\hline Intercept & 233 & $* 9.76$ E-06 & 52 & 3.8 E-05 \\
& & $($ significant) & & (significant) \\
\hline
\end{tabular}

Note: *Statistically significant $(p<0.05)$

$(p>0.05) .{ }^{24}$ Model $\mathrm{F}$ values for MPS and EE were 52.78 and 16.78, respectively, while their corresponding $F_{\text {tab }}$ values were 3.68 and 3.14 , respectively, which implies that the models are highly significant ( $p$-value $<0.05)$. However, when the coefficients of the two independent variables in equation 4 were compared, the value for $\mathrm{X}_{2}$ $\left(b_{2}=-28.33\right)$ was found to be maximum and hence it was considered to be a major contributing factor affecting the particle size of the MTX loaded NLCs. Here, the negative sign indicates the increase in the level of one variable causing a decrease in the response parameter, while a positive sign indicates the increase in the level of one variable causing an increase in the respective response parameter. It was found that the change in the ratio of Compritol 888 and Capmul MCM had a significant effect on the particle size. Similarly, the value for $\mathrm{X}_{1}\left(\mathrm{~b}_{1}=4.5\right)$ as shown in equation 5 was also highest the between two independent variables, indicating a major contributory factor as a variable for EE. However, a quadratic term of $\mathrm{X}_{2}\left(\mathrm{X}_{22}\right)$ was found to be maximum $(-7.5)$, pointing to a negative influence on the $\mathrm{EE}$ due to interaction between $\mathrm{X}_{2}$. The goodness of fit of the model was checked by the determination coefficient $\left(\mathrm{R}^{2}\right)$.

The values of $\mathrm{R}^{2}$ for MPS (0.9887) and EE (0.965) for the full model indicated that over $98 \%$ of the total variations were explained by the model. The values of adjusted determination coefficients $\left(\operatorname{adj} \mathrm{R}^{2}\right.$ ) were also very high ( 0.97 for MPS and 0.91 for EE) for the full model, indicating the significance of the model because $\mathrm{R}^{2}$ value near to 1 signifies an excellent correlation between the independent variables. Thus, all the above considerations indicate that the model was highly significant and can explain $99 \%$ variations around the mean value.

\section{Contour Plots and Surface Response Curves}

The contour plot shows predicted MPS values at different levels of Compritol 888 and Capmul MCM (Figure 1). It can be seen that an increase in the amount of Compritol 888 increased MPS, while increasing the Capmul MCM amount led to a decrease in the MPS. The surface response curve also shows increased particle size with an increasing amount of solid lipid. The size of lipid nanoparticles is highly dependent on lipid concentration, which can be explained in terms of the tendency of lipid to coalesce at high lipid concentration. According to Stoke's law, this behavior can be explained by the difference in density between the internal and external phases. ${ }^{32}$ In contrast, an increase in the amount of liquid lipid showed a reduction in particle size. The slight curvature in the surface response curve points towards the insignificance of quadratic effects. $\left(\mathrm{X}_{2}^{2}\right)$. 
A

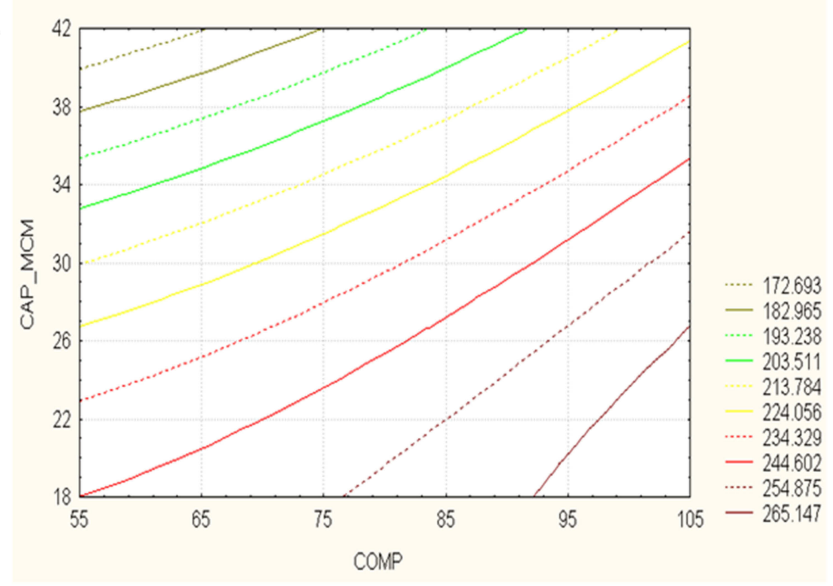

C

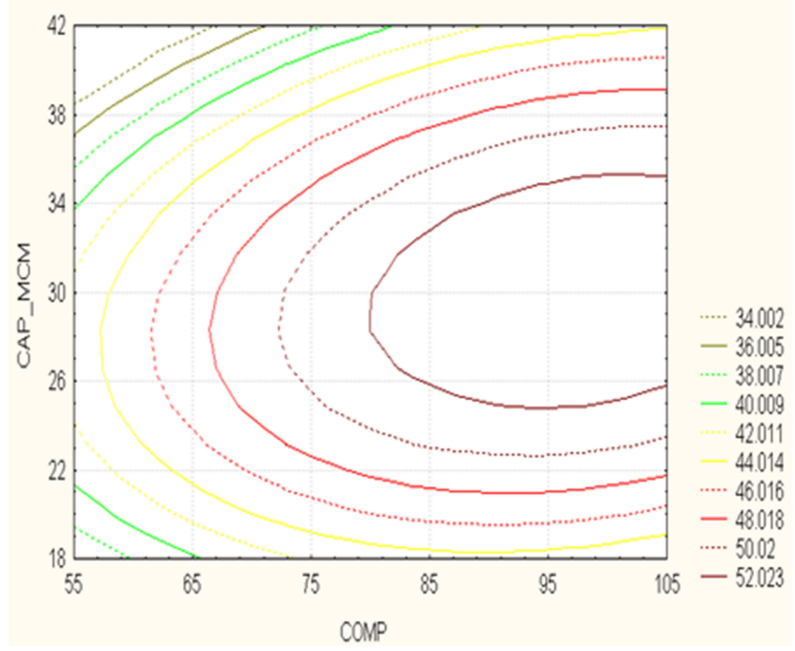

B

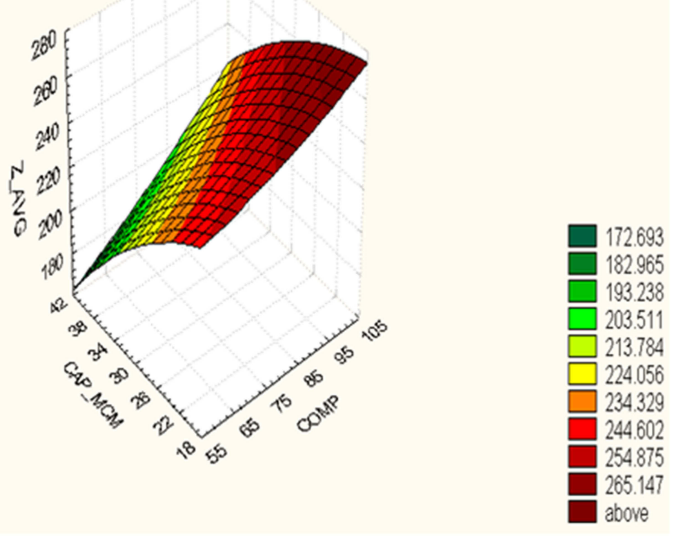

D

Figure I (A) Contour plot of Zavg versus the amount of Compritol 888 and Capmul MCM; (B) response surface plot of Zavg versus the amount of Compritol 888 and Capmul MCM; (C) the contour plot of \% drug entrapment vs the amount of Compritol 888 and Capmul MCM; (D) response surface plot of \% drug entrapped vs the amount of Compritol 888 and Capmul MCM.

However, we have not studied the effect of Capmul MCM below $18 \mathrm{mg}$ and hence it would be difficult to conclusively predict the significance of the quadratic effects. Figure 1 reveals an increase in $\mathrm{EE}$ when the amount of Compritol 888 was increased up to $105 \mathrm{mg}$, with a fixed amount of Capmul MCM (30 mg), whereas the particle size was found to be increased beyond $100 \mathrm{mg}$. Similarly, EE was found to be increased when the amount of Capmul MCM (liquid lipid) was increased up to $30 \mathrm{mg}$, while decreased entrapment was observed beyond $30 \mathrm{mg}$ of liquid lipid. This may be due to lipid precipitation during particle formation. After homogenization, the mixture of drug and lipid in each droplet is cooled, which leads to precipitation of the lipid earlier than the drug; this ultimately results in a drug-free core or at least a core with reduced drug content. ${ }^{33}$ Thus, an increase in the lipid load beyond a certain extent (as optimized) causes the drug to be present in the outer shell or remain outside the NLC in the external aqueous phase leading to poor entrapment efficiency. This can be seen by the presence of curvature in the surface response curve for EE indicating the significance of quadratic terms $\left(\mathrm{X}_{1}{ }^{2}\right.$ and $\left.\mathrm{X}_{2}{ }^{2}\right)$ (Figure 1). Thus, it can be concluded that optimum responses (minimum possible PS and maximum possible EE) can be attained when the amount of Compritol 888 and Capmul MCM was 60-100 $\mathrm{mg}$ and 20-40 mg, respectively.

\section{Characterization of NLCs Morphology}

Results of the TEM analysis of NLCs indicated rod-shaped NLCs (Figure 4) with narrow size distribution and were non-aggregated. The diameters of the particles observed in 
the micrographs are in good agreement with the data obtained from the Malvern particle size analyzer (Table 1).

\section{Differential Scanning Calorimetry}

DSC gives information regarding the crystalline or amorphous nature of the samples. Figure 2 shows DSC curves of pure MTX, Compritol 888, physical mixture, and lyophilized MTXNLCs. Thermograms of bulk and Compritol 888 exhibited endothermic peaks at $136.54^{\circ} \mathrm{C}$ and $72.81^{\circ} \mathrm{C}$, respectively, corresponding to their melting temperatures (Figure 2A and B). The peak for MTX was completely absent in lyophilized MTXNLCs (Figure 2D), while it was clearly evident in the physical mixture of MTX $\left(136.54^{\circ} \mathrm{C}\right)$ and Compritol $888\left(71.24^{\circ} \mathrm{C}\right)$ as shown in Figure 6C. It has been reported that when the drug does not show its endothermic peak in the nanoparticulate formulations, it is in the amorphous state. ${ }^{33,34}$ Hence, it could be concluded that the drug was present in the amorphous phase and may have been homogeneously dispersed in the NLCs.

\section{X-Ray Diffraction}

The XRD data of pure MTX clearly exhibited its highly crystalline nature with a principal peak at $2 \theta$ value of 27.304, whereas Compritol 888 showed a principal peak at $2 \theta$ value of 21.226 (Figure $3 \mathrm{~A}$ and $\mathrm{C}$ ). In the physical

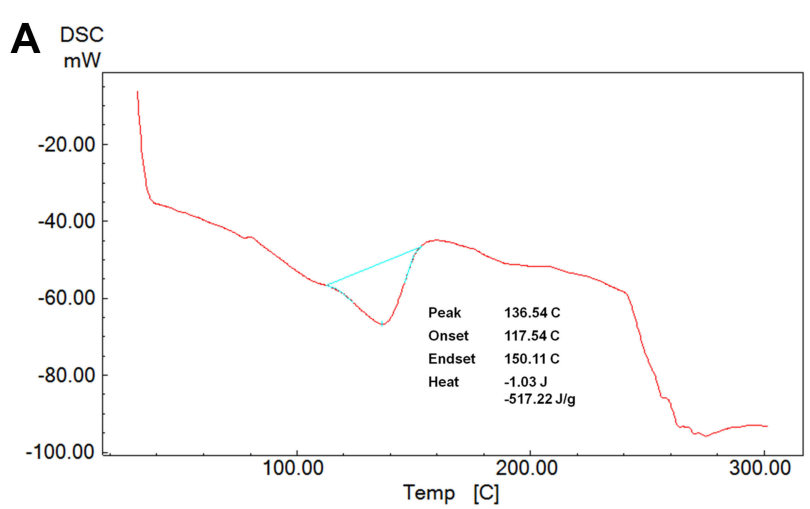

B

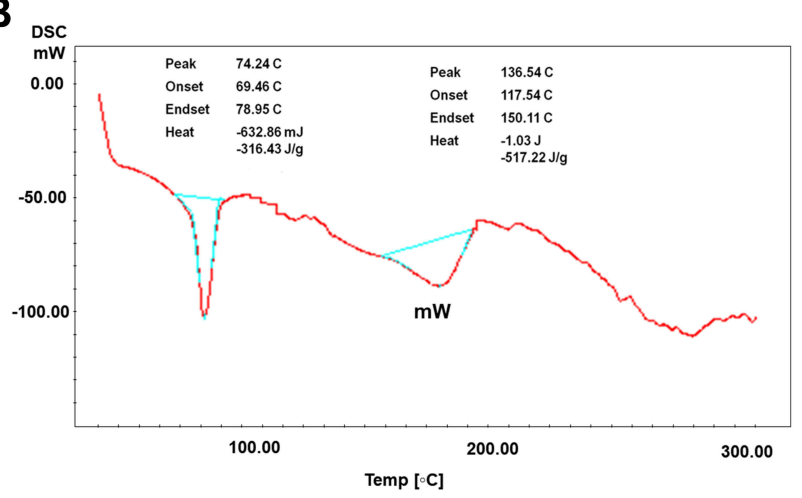

mixture of MTX and Compritol 888, the crystalline peak for Compritol 888 was clearly evident at $2 \theta$ value of 21.27 (Figure 3D), whereas no peak for MTX (at $2 \theta$ value of 27.304) was observed in the NLC formulation, indicating its presence in an amorphous or molecularly dispersed state. The XRD of lyophilized MTXNLCs showed some crystalline modification in Compritol 888, indicating disorderliness of the crystalline structure of solid lipid due to liquid lipid. Moreover, XRD of the cryoprotectant (trehalose dehydrate) (Figure 3B) alone confirmed that the peak at $2 \theta$ value of 20 in the NLC may be due to trehalose dehydrate. $^{35}$

\section{Evaluation of MTX Loaded NLCs In vitro Release Studies}

The cumulative percentage release of methotrexate from MTX suspension (MTX-S) and MTXNLCs was investigated over a period of 5 and $30 \mathrm{~h}$ respectively. Each sample was analyzed in triplicate and release curves are shown in Figure 4. It was observed that MTX-S released $82.15 \pm 4.69 \%$ of the drug at the end of $5 \mathrm{~h}$, while MTXNLCs showed a $30.05 \pm 0.50 \%$ drug release at $10 \mathrm{~h}$. Furthermore, MTXNLCs showed $61.91 \pm 2.22 \%$ of MTX release with sustained and steady release over the entire

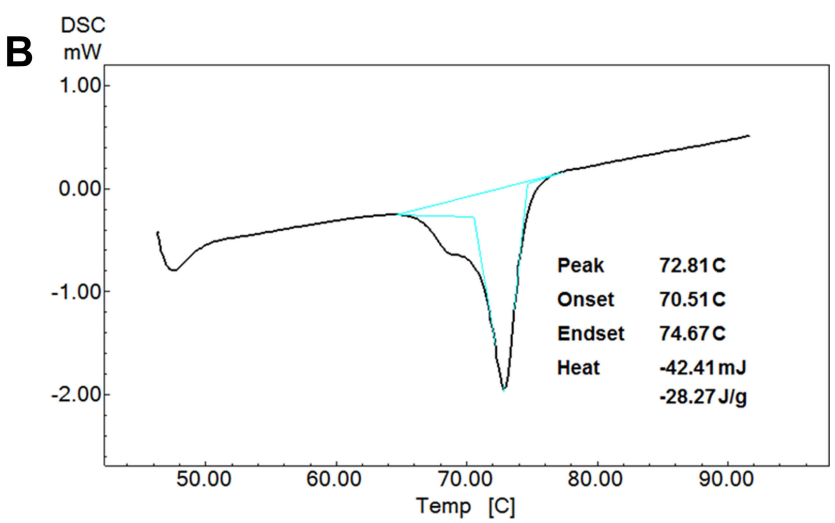

D

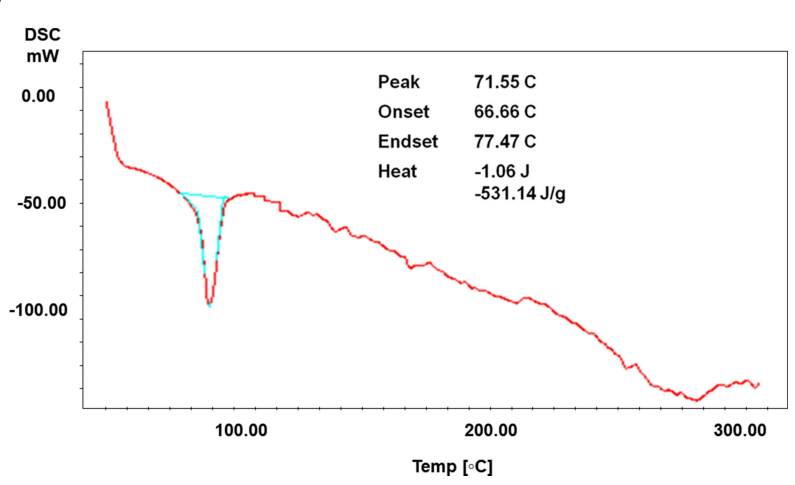

Figure 2 DSC thermograms of (A) methotrexate; (B) Compritol 888; (C) physical mixture of methotrexate and Compritol 888; (D) MTXNLC formulation. 

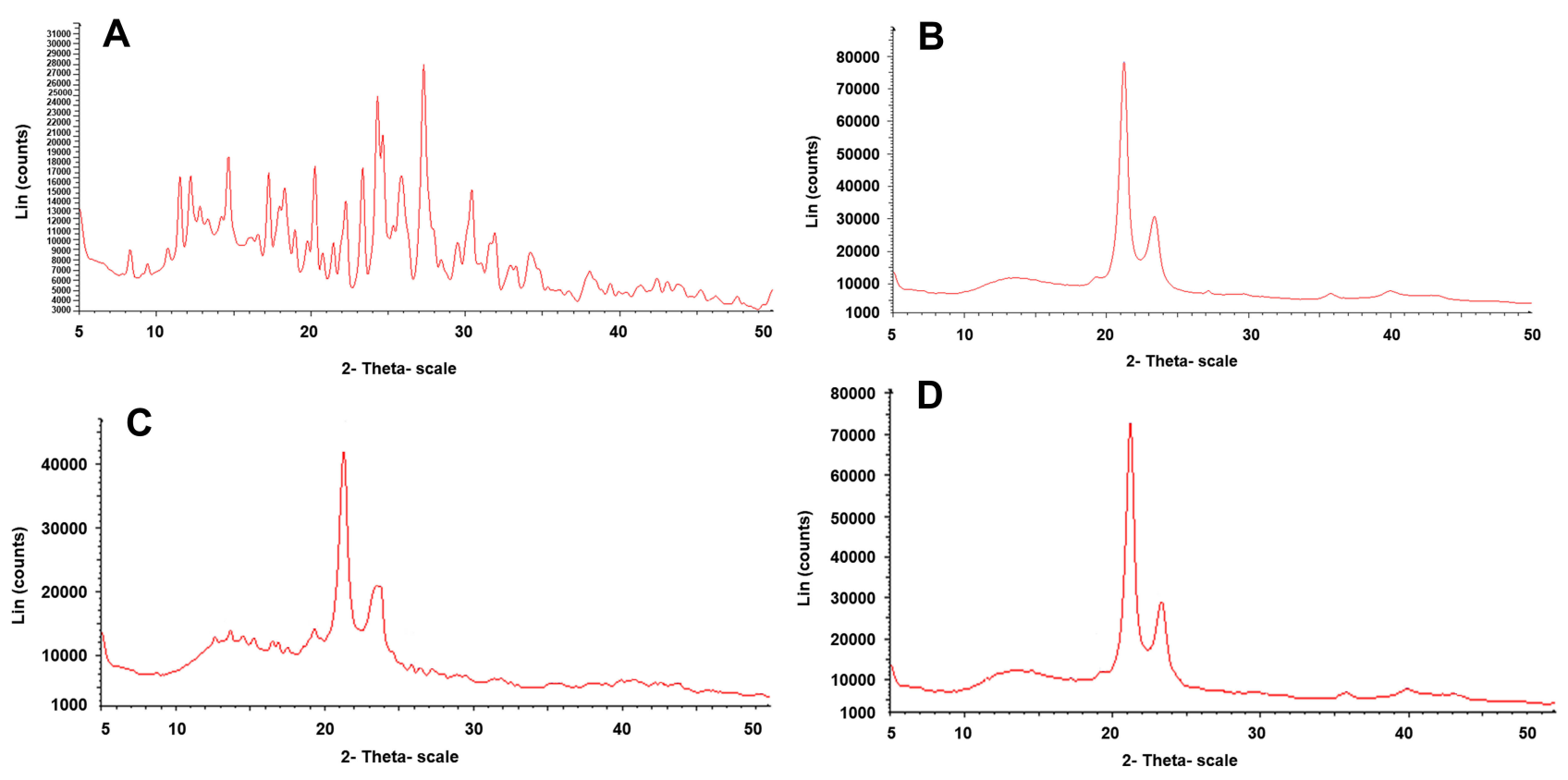

Figure 3 XRD of (A) methotrexate; (B) Compritol 888; (C) physical mixture of Compritol 888 and methotrexate; and (D) lyophilized MTXNLC formulation.

period of study $(30 \mathrm{~h})$. Relatively slow and sustained drug release was observed from the MTXNLC formulation, which can be attributed to the penetration of the aqueous diffusion medium into the hydrophobic lipid followed by slow dissolution and diffusion of the drug from the NLCs. The drug released from the lipid matrix by zero-order diffusion. This kind of drug release pattern is of interest in dermal application, as sustained release provides the drug over a prolonged period of time with improved penetration into the deeper layer of the skin. In comparison to the drug release from MTX suspension showing more than $80 \%$ drug release within $5 \mathrm{~h}$, MTXNLCs present a better delivery system for MTX.

The drug release data from the MTXNLCs were fitted into the Korsemeyers Peppas model to find the release mechanism. The release exponent " $n$ " of MTXNLCs was 0.53 , showing a combination of dissolution and diffusion mechanism (anomalous diffusion $-0.5<\mathrm{n}<1$ ) with $\mathrm{R}^{2}$ value of 0.992 ; this may indicate that the drug release is controlled by matrix diffusion-based kinetics. ${ }^{36}$

\section{In vitro Skin Deposition Studies}

In vitro skin deposition of plain MTX gel and MTXNLC gel was investigated using HCS and the results are compared in Figure 4C. The study revealed a significantly higher deposition of MTX from MTXNLCs gel in the skin $(71.52 \pm 1.13 \%)$ than the plain MTX gel $(38.48$ $\pm 0.96 \%$ ). The results of these studies supported the hypothesis that the incorporation of MTX into NLC enhances the drug deposition into HCS. The results are also in agreement with the reports that SLN and NLCs improve the dermal localization of several topical therapeutic agents. ${ }^{37,38}$ One of the reasons to employ the NLC approach for topical delivery of MTX was to improve its dermal localization for reducing its systemic toxicity and enhancing the localized treatment of psoriasis.

\section{Effect of MTX Loaded NLCs in IMQ-Induced Psoriasis}

The effect of the MTXNLC gel in the attenuation of psoriasis symptoms was evaluated using IMQ-induced psoriasis in a mouse model. IMQ-induced psoriasis is a widely used animal model for the study of the efficacy of various drugs and formulations in experimental psoriasis. ${ }^{39}$ The MTXNLC gel exhibited significant anti-psoriasis activity as compared to the control and plain MTX-gel formulation.

\section{Effect of MTXNLCs on Ear Thickness}

The topical application of IMQ to the mice ear resulted in the increased thickening of dorsal skin lesions from day 0 to day 6. Ear thickness in mice was assessed as an index of skin inflammation. Ear thickness of the mice was measured as an independent parameter of skin inflammation. ${ }^{25}$ The IMQ plus MTXNLC treated group showed significantly lower thickness values as compared to the IMQtreated group. However, the MTX plain gel inhibited the thickening of mice skin, but the results are less prominent 

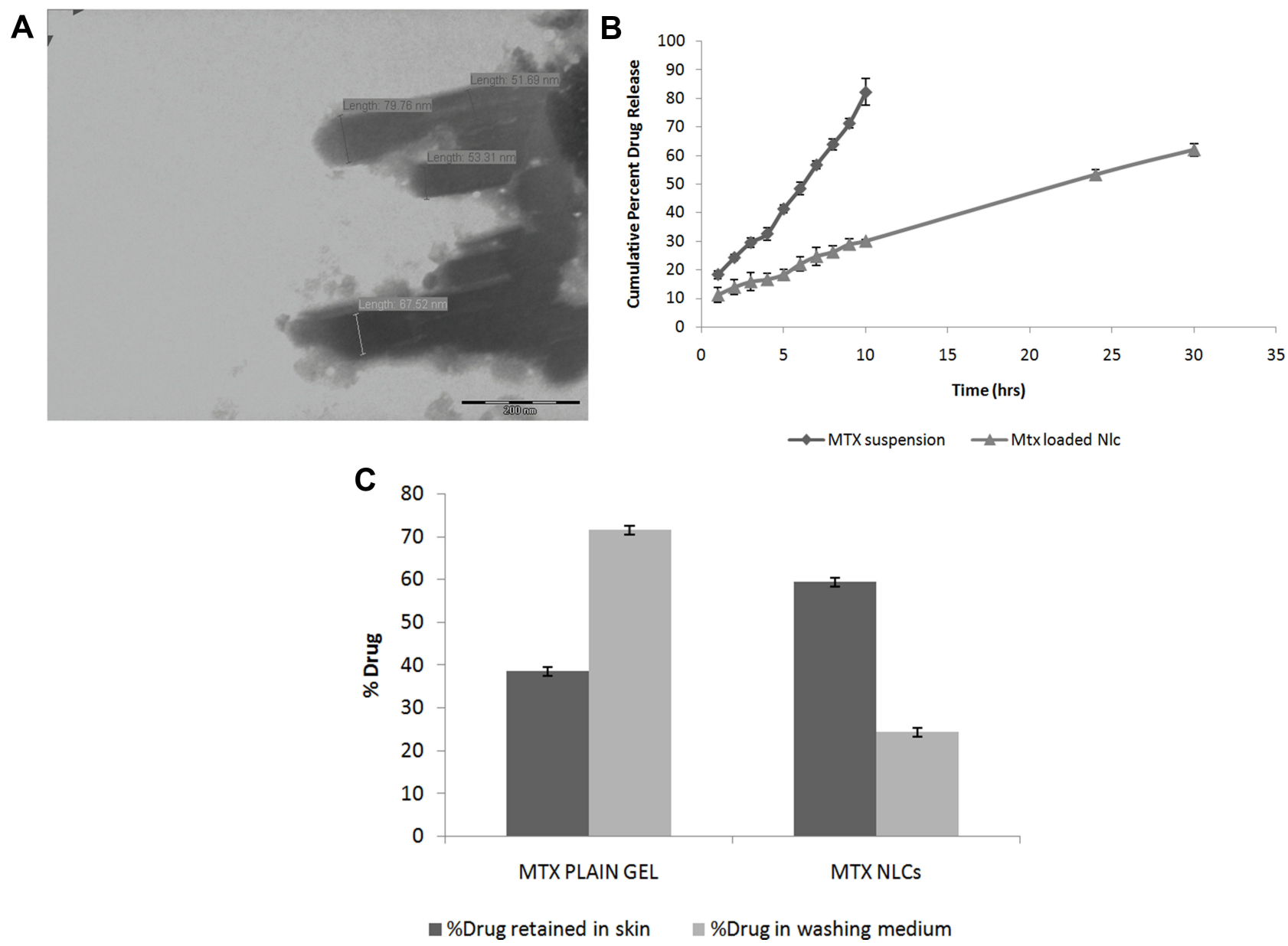

Figure 4 (A) TEM of the MTXNLCs; (B) cumulative percent drug release of methotrexate in phosphate buffer pH 7.4; (C) in vitro skin deposition study of MTX plain gel and NLCs.

as compared to the inhibition of ear thickness by MTXNLC formulation, as shown in Figure 5.

\section{Effect of MTXNLCs on Morphological Features in IMQ-Induced Psoriasis in Mice}

To assess the effect of MTXNLCs on morphological features of IMQ-induced psoriasis we used a PASI scoring system. The PASI scores include skin thickness, erythema, and scaling. IMQ application resulted in increased PASI scores due to increased thickness, erythema, and scaling. As compared to the normal animal, a significant increase in the PASI score was noted in the IMQ-treated group. PASI score was significantly $(p<0.001)$ attenuated in MTXNLCs and plain MTX gel-treated groups (Figure 6). The effect of MTXNLCs $\left(100 \mu \mathrm{g} / \mathrm{cm}^{2}\right)$ was found to be comparatively effective with the plain MTX gel. The effect was pronounced due to the better release of MTX from MTXNLCs and increases in bioavailability.

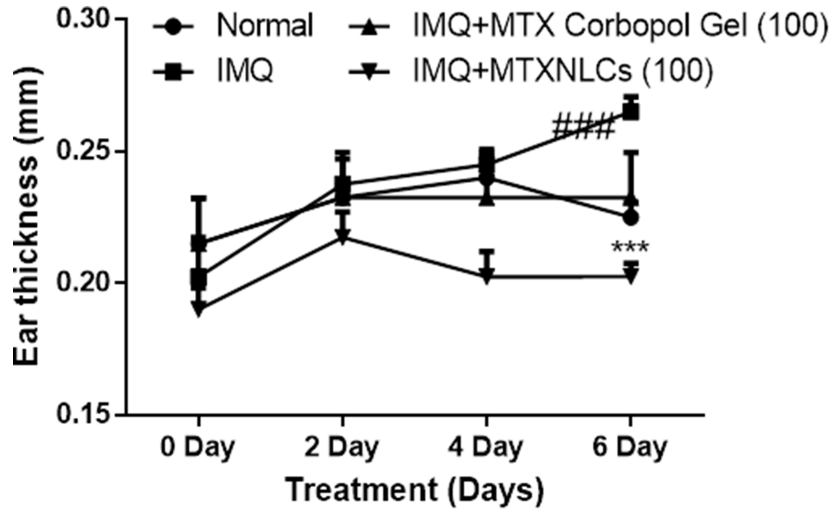

Figure 5 Effect of MTXNLCs on ear thickness in IMQ-induced psoriasis. Data are analysed by two way ANOVA and represented as mean \pm SEM.Post-test-Bonferroni posthoc test. $p<0.05$ is con- sidered statistically significant. $* * * p<0.001$ is compared with the IMQ control group and \#\#p $<0.001$ is compared with normal.

\section{Effect of MTXNLCs on Organ Weights}

IMQ application in the mice resulted in the enlargement of the spleen and increased spleen weight. The spleen weight 

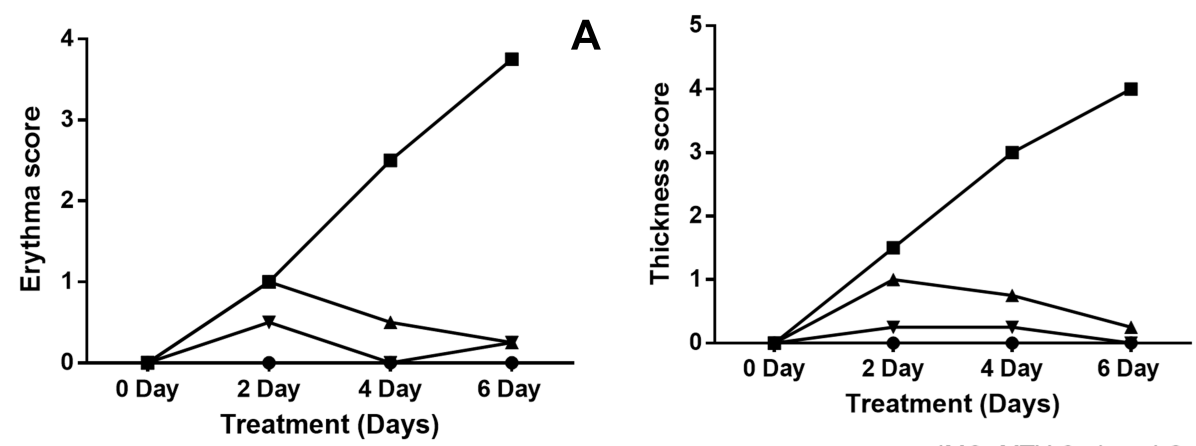

\section{B}
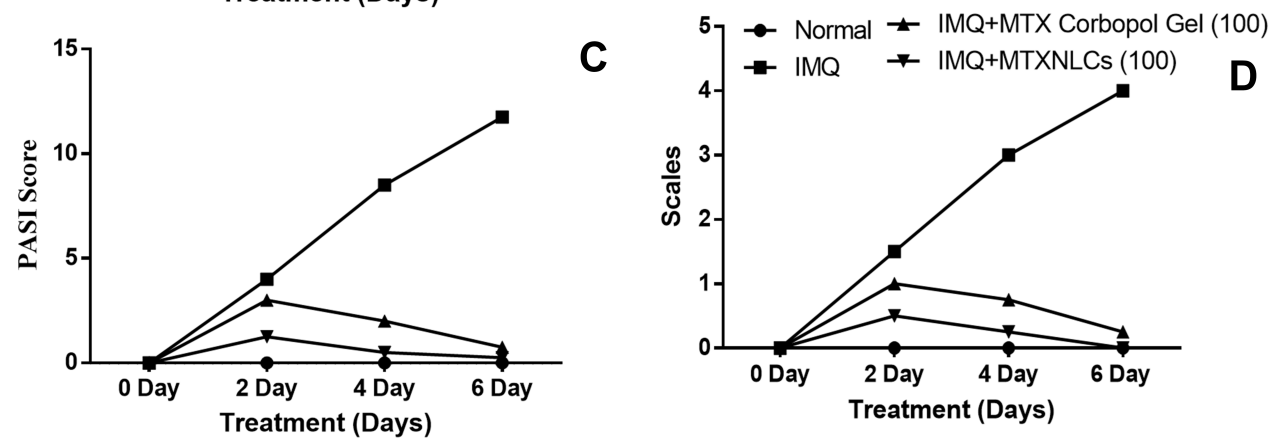

Figure 6 Effect of MTXNLCs on morphological features in IMQ-induced psoriasis in mice. (A) Erythema score; (B) thickness score; (C) PASI score; (D) scaling score. Post-test-Kruskal-Wallis test. $p<0.05$ was considered statistically significant.

was significantly reduced in the animals receiving the topical application of MTXNLCs at the dosage of 100 $\mu \mathrm{g} / \mathrm{cm}^{2}$ and as compared to the animals treated with only IMQ. Reduction of spleen weight following treatment with MTXNLCs in IMQ-induced psoriasis in mice indicates the diminution of humoral immune responses. ${ }^{40}$ Similarly, we observed significant $(p<0.001)$ decreases in the weight of thymus and lymph node in MTXNLC-treated animals as compared to IMQ-treated mice (Table 3).

\section{Effect of MTXNLCs on SOD, GSH, CAT, and MDA Levels}

We measured the SOD, GSH, CAT, and MDA levels in mouse skin samples by respective assay kits. We noted a significant reduction of CAT, GSH, and SOD levels as well as increased levels of MDA in IMQ-treated animals as compared to the normal animals. The topical application of MTXNLCs at the dosage of $100 \mu \mathrm{g} / \mathrm{cm}^{2}$ and MTX plain gel $\left(100 \mu \mathrm{g} / \mathrm{cm}^{2}\right)$ resulted in the significant ( $p<0.001$ and $p<0.01$, respectively) elevation of GSH, CAT, and SOD activities. Moreover, the treatment of mice with MTX loaded NLCs or with plain MTX gel showed suppression of MDA levels in mouse skin samples (Table 4). These results imply that MTXNLCs were able to maintain the balance between oxidative and antioxidative systems in an experimental model of IMQinduced psoriasis. Oxidative stress has an important role in the development and progression of various skin diseases including psoriasis. ${ }^{41}$ Reactive oxygen species can stimulate various inflammatory pathways including MAPKs and NF- $\kappa B$ which lead to inflammatory pathology. ${ }^{42}$ The MTXNLCs showed better protection as

Table 3 Effect of MTXNLCs on Organ Weight in IMQ-Induced Psoriasis

\begin{tabular}{|l|l|l|l|l|}
\hline \multirow{2}{*}{ Group } & \multicolumn{2}{l|}{ Organ Weight (mg) } & \multicolumn{2}{l|}{ Spleen } \\
\cline { 2 - 5 } & Ear Weight & Lymph Node & $64 \pm 14$ & Thymus \\
\hline Normal & $3.3 \pm 0.48$ & $24 \pm 3.9$ & $166 \pm 10^{\# \#}$ & $50 \pm 6.6$ \\
IMQ & $5.0 \pm 0.41^{\# \#}$ & $30 \pm 2.0^{\# \#}$ & $159 \pm 12^{* * *}$ & $60 \pm 4.7^{\# \#}$ \\
IMQ+MTX Carbopol gel (100) & $3.3 \pm 0.48^{* * *}$ & $24 \pm 1.5^{* * *}$ & $108 \pm 10^{* * *}$ & $48 \pm 3.7^{* * *}$ \\
IMQ+MTXNLCs (100) & $3.0 \pm 0.41^{* * *}$ & $18 \pm 1.9^{* * *}$ & $37 \pm 4.6^{* * *}$ \\
\hline
\end{tabular}

Notes: Data were represented as mean \pm SEM. Post-test Bonferroni post hoc test. ${ }^{* * *} p<0.00 \mathrm{I}$ as compared with the IMQ and ${ }^{\# \#} p<0.00 \mathrm{I}$ is compared with normal. 
Table 4 Effect of MTXNLCs on Lipid Peroxidation, Gluththione and Antioxidant Enzymes

\begin{tabular}{|l|l|l|l|l|}
\hline Group & $\begin{array}{l}\text { MDA } \\
(\mu \mathrm{g} / \mathrm{mg} \text { of Protein) }\end{array}$ & $\begin{array}{l}\text { GSH } \\
(\mu \mathrm{g} / \mathrm{mg} \text { of Protein) }\end{array}$ & $\begin{array}{l}\text { CAT } \\
\text { (U/mg of Protein) }\end{array}$ & $\begin{array}{l}\text { SOD } \\
\text { (U/mg of Protein) }\end{array}$ \\
\hline Normal & $39 \pm 4.6$ & $40 \pm 1.4$ & $34 \pm 3.8$ & $28 \pm 1.8$ \\
IMQ & $112 \pm 25^{\# \#}$ & $9.8 \pm 1.8^{\# \#}$ & $13 \pm 2.2^{\# \#}$ & $15 \pm 3.4^{\# \#}$ \\
IMQ+MTX Carbopol gel (100) & $52 \pm 13^{* * *}$ & $17 \pm 2.5^{* * *}$ & $23 \pm 5.4^{* * *}$ & $20 \pm 4.7^{* * *}$ \\
IMQ+MTXNLCs (100) & $37 \pm 8.8^{* * *}$ & $37 \pm 5.5^{* * *}$ & $31 \pm 3.6^{* * *}$ & $26 \pm 1.5^{* * *}$ \\
\hline
\end{tabular}

Notes: Data were represented as mean \pm SEM. Post-test- Bonferroni post hoc test. ${ }^{* * *} p<0.00 \mathrm{I}$ as compared with the IMQ and ${ }^{\# \# \#} p<0.00 \mathrm{I}$ as compared with normal.

compared to MTX plain gel. The NLC formulation increases bioavailabilit and hence showed better activity as compared to MTX plain gel.

\section{Effect of MTXNLCs on Cytokine Levels}

Inflammatory cytokines including TNF- $\alpha$, IL-1 $\beta$, and IL-6 were measured in the skin samples of normal, IMQ-applied, IMQ + MTX plain gel treated and IMQ + MTX loaded NLC formulation treated mice by ELISA assay. The expression of TNF- $\alpha$, IL-1 $\beta$, and IL- 6 was found to be significantly increased in skin samples of IMQ-applied mice as compared to the normal mice. Meanwhile, a significant decrease in the expression of all three measured cytokines (TNF- $\alpha$, IL-1 $\beta$, and IL-6) was noted following the application of MTXNLCs at $100 \mu \mathrm{g} / \mathrm{cm}^{2}$. The treatment of mice with plain MTX gel also resulted in a decrease in cytokine levels (Figure 7). A marked decrease in the cytokine levels by the MTXNLCs implies the protective effect of tested MTXNLCs against IMQ-induced inflammatory lesions in mice.

\section{Effect of MTXNLCs on Histopathological Alterations}

IMQ-induced histopathological alterations in the mice ear samples were observed using hematoxylin and eosin stain. Inflammatory cell infiltration and increased epithelial thickness were observed in the IMQ-treated group as compared to normal animals. The hematoxylin and eosin staining of the ear samples revealed increased parakeratosis, acanthosis, and inflammatory cell infiltration. The treatment of animals with MTXNLCs showed significant attenuation of histological alterations. The effect observed in the MTXNLC group was better than the effect observed in the plain MTX-gel group (Figure 8). ${ }^{43}$

\section{Conclusion}

The formulation of MTXNLCs was optimized using a $3^{2}$ factorial experimental design, selecting the solid lipid and liquid lipid contents for achieving optimum particle size and maximum entrapment efficiency. The resultant NLCs were smooth as seen under TEM. DSC and XRD study indicated an amorphous or molecularly dispersed state of MTXNLCs. The in vitro release study of MTXNLC showed sustained release of drug for $30 \mathrm{~h}$. In vitro skin deposition studies showed significantly higher $(p<0.05)$ deposition of MTX from MTXNLCs gel in the skin than plain MTX gel. The findings of this investigation conclusively demonstrate the promising role of MTXNLC gel in the treatment of psoriasis.
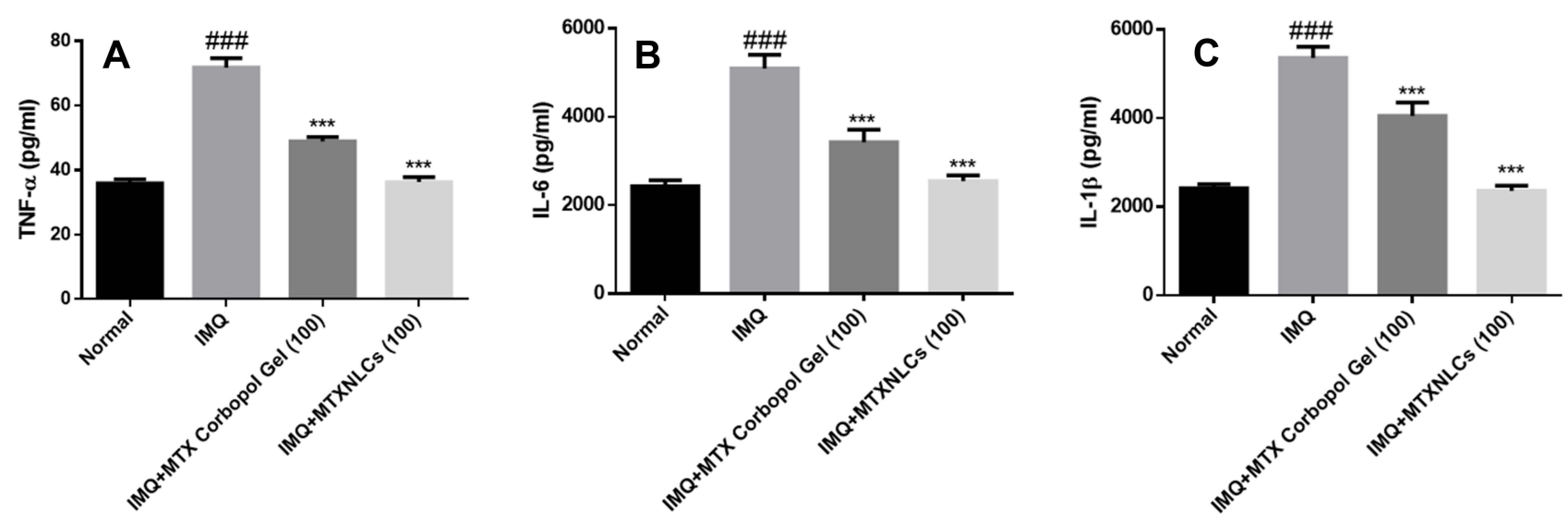

Figure 7 Effect of MTXNLCs on the release of cytokines in IMQ-induced psoriasis in mice. (A) TNF- $\alpha$; (B) IL-6; (C) IL-I $\beta$. Data are represented as mean \pm SEM. Post-testBonferroni post hoc test. ${ }^{* * *} p<0.001$ as compared with the IMQ and ${ }^{\# \#} p<0.001$ is compared with normal. 


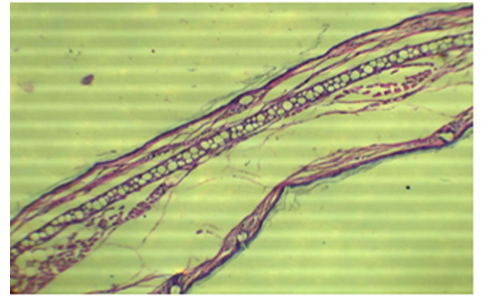

Normal

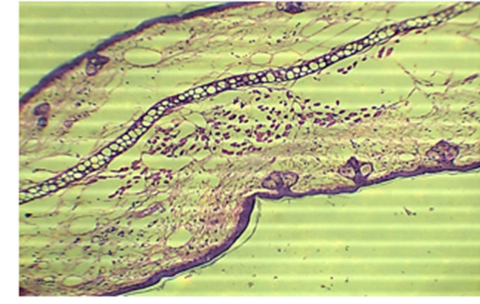

IMQ

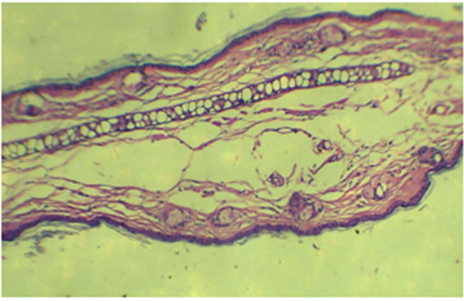

IMQ+MTXNLCs (100)

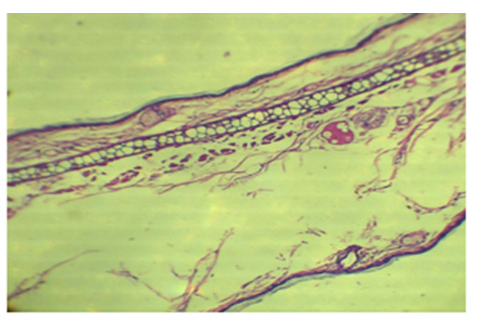

IMQ+MTX Carbopol gel (100)

Figure 8 Effect of MTXNLCs on histopathological alterations in IMQ-induced psoriasis in mice.

Apart from improving the therapeutic response, NLC based MTX gel also caused a reduction in adverse effects, fostering better patient compliance.

The evaluation of MTX loaded NLCs in the IMQinduced psoriasis model displayed significant antipsoriasis activity. The developed MTXNLCs decrease the PASI score, oxidative stress, inflammatory cytokines, organ weights, and protected the animals from IMQ-induced histopathological alterations in mouse skin. The MTXNLCs showed more promising effects, as compared to the plain MTX-gel formulation which might be due to an increase in the bioavailability and contact time of the drug. Thus, MTXNLC gel may be considered as an alternative to existing therapy of psoriasis using available formulations in the market. However, the role of NLC-based gel formulations may only be established after clinical evaluation of a large number of patients, with a special focus on the adverse symptoms of therapy with extensive toxicological studies.

\section{Acknowledgments}

The authors gratefully acknowledge the financial support received from DBT- Bio-CARe, New Delhi, India to Dr. Yogeeta O. Agrawal. The authors also acknowledge the financial support to the research works in the laboratory of Dr. Shreesh Ojha from United Arab Emirates University, United Arab Emirates.

\section{Disclosure}

The authors report no conflicts of interest in this work.

\section{References}

1. Christophers E. Psoriasis- epidemiology and clinical spectrum. Clin Exp Dermatol. 2001;26:314-320. doi:10.1046/j.1365-2230.2001.00832.x

2. Pradhan M, Alexander A, Singh MR, Singh D, Saraf S, Saraf S. Understanding the prospective of nano-formulations towards the treatment of psoriasis. Biomed Pharmacotherap. 2018;107:447-463. doi:10.1016/j.biopha.2018.07.156

3. Gisondi P, Del Giglio M, Girolomoni G. Treatment approaches to moderate to severe psoriasis. Int J Mole Sci. 2017;18:2427.

4. Gungor S, Rezigue M. Nanocarriers mediated topical drug delivery for psoriasis treatment. Curr Drug Metab. 2017;18:454-468. doi: $10.2174 / 1389200218666170222145240$

5. Tapeinos C, Battaglini M, Ciofani G. Advances in the design of solid lipid nanoparticles and nanostructured lipid carriers for targeting brain diseases. J Control Rel. 2017;264:306-332. doi:10.1016/j.jconrel.2017.08.033

6. Jenning V, Gysler A, Schäfer-Korting M, Gohla SH. Vitamin A loaded solid lipid nanoparticles for topical use: occlusive properties and drug targeting to the upper skin. Eur J Pharm Biopharm. 2000;49:211-218. doi:10.1016/S0939-6411(99)00075-2

7. Kamel AE, Fadel M, Louis D. Curcumin-loaded nanostructured lipid carriers prepared using Peceol $^{\mathrm{TM}}$ and olive oil in photodynamic therapy: development and application in breast cancer cell line. Int J Nanomed. 2019;14:5073. doi:10.2147/IJN.S210484

8. Jenning V, Gohla SH. Encapsulation of retinoids in solid lipid nanoparticles (SLN). J Microencapsul. 2001;18(2):149-158. doi:10.1080/ 02652040010000361

9. Souto E, Wissing S, Barbosa C, Müller R. Development of a controlled release formulation based on SLN and NLC for topical clotrimazole delivery. Int J Pharm. 2004;278:71-77. doi:10.1016/j. ijpharm.2004.02.032

10. Küchler S, Herrmann W, Panek-Minkin G, et al. SLN for topical application in skin diseases - Characterization of drug-carrier and carrier-target interactions. Int $J$ Pharm. 2010;390:225-233. doi:10.1016/j.ijpharm.2010.02.004 
11. Tung N-T, Vu V-D, Nguyen P-L. DoE-based development, physicochemical characterization, and pharmacological evaluation of a topical hydrogel containing betamethasone dipropionate microemulsion. Colloids Surf B. 2019;181:480-488. doi:10.1016/j. colsurfb.2019.06.002

12. Borgia SL, Regehly M, Sivaramakrishnan R, et al. Lipid nanoparticles for skin penetration enhancement-correlation to drug localization within the particle matrix as determined by fluorescence and parelectric spectroscopy. J Control Rel. 2005;110:151-163. doi:10.1016/j.jconrel.2005.09.045

13. Li Q, Cai T, Huang Y, Xia X, Cole S, Cai Y. A review of the structure, preparation, and application of NLCs, PNPs, and PLNs. Nanomaterial. 2017;7:122. doi:10.3390/nano7060122

14. Kaul S, Gulati N, Verma D, Mukherjee S, Nagaich U. Role of nanotechnology in cosmeceuticals: a review of recent advances. J Pharm. 2018;2018.

15. Souto EB, Muller R. Lipid nanoparticles (solid lipid nanoparticles and nanostructured lipid carriers) for cosmetic, dermal, and transdermal applications. Drugs Pharmaceut Sci. 2007;166:213.

16. Hollywood A, O'Keeffe C, Boggs J, Feighery C, Collins S. The utility of subcutaneous methotrexate for chronic plaque psoriasis in a real-world setting. Brit J Dermatol. 2019.

17. Costa P, Lobo JMS. Modeling and comparison of dissolution profiles. Eur J Pharm Sci. 2001;13:123-133. doi:10.1016/S0928-0987(01) 00095-1

18. Harrison C, Jenski L, Voychehovski T, Bernstein D. Modification of immunological responses and clinical disease during topical R-837 treatment of genital HSV-2 infection. Antiviral Res. 1988;10:209-223. doi:10.1016/0166-3542(88)90032-0

19. Hejri A, Khosravi A, Gharanjig K, Hejazi M. Optimisation of the formulation of $\beta$-carotene loaded nanostructured lipid carriers prepared by solvent diffusion method. Food Chem. 2013;141 (1):117-123. doi:10.1016/j.foodchem.2013.02.080

20. Mehta AK, Yadav KS, Sawant KK. Nimodipine loaded PLGA nanoparticles: formulation optimization using factorial design, characterization and in vitro evaluation. Curr Drug Del. 2007;4:185-193. doi:10.2174/156720107781023929

21. Singka GSL, Samah NA, Zulfakar MH, Yurdasiper A, Heard CM. Enhanced topical delivery and anti-inflammatory activity of methotrexate from an activated nanogel. Eur $J$ Pharm Biopharm. 2010;76:275-281. doi:10.1016/j.ejpb.2010.06.014

22. Tofani R, Sumirtapura Y, Darijanto S. Formulation, characterisation, and in vitro skin diffusion of nanostructured lipid carriers for deoxyarbutin compared to a nanoemulsion and conventional cream. Scientia Pharma. 2016;84:634-645. doi:10.3390/scipharm84040634

23. Han F, Yin R, Che X, et al. Nanostructured lipid carriers (NLC) based topical gel of flurbiprofen: design, characterization and in vivo evaluation. Int J Pharm. 2012;439:349-357. doi:10.1016/j.ijpharm.2012.08.040

24. Parmar SJ, Easwaran S. Metronidazole-loaded nanostructured lipid carriers to improve skin deposition and retention in the treatment of rosacea. 2019.

25. van der Fits L, Mourits S, Voerman JS, et al. Imiquimod-induced psoriasis-like skin inflammation in mice is mediated via the IL-23/IL-17 axis. J Immunol. 2009;182(9):5836-5845. doi:10.4049/jimmunol.08 02999

26. Sun Y, Zhang J, Huo R, et al. Paeoniflorin inhibits skin lesions in imiquimod-induced psoriasis-like mice by downregulating inflammation. Int Immunopharmacol. 2015;24:392-399. doi:10.10 16/j.intimp.2014.12.032

27. Schaper K, Dickhaut J, Japtok L, et al. Sphingosine-1-phosphate exhibits anti-proliferative and anti-inflammatory effects in mouse models of psoriasis. J Dermatol Sci. 2013;71(1):29-36. doi:10. 1016/j.jdermsci.2013.03.006
28. Arora N, Shah K, Pandey-Rai S. Inhibition of imiquimod-induced psoriasis-like dermatitis in mice by herbal extracts from some Indian medicinal plants. Protoplasma. 2016;253:503-515. doi:10.1007/ s00709-015-0829-y

29. Molls RR, Savransky V, Liu M, et al. Keratinocyte-derived chemokine is an early biomarker of ischemic acute kidney injury. Am J Physiol-Renal Physiol. 2006;290(5):F1187-F1193. doi:10.1152/ ajprenal.00342.2005

30. Gore PR, Prajapati CP, Mahajan UB, et al. Protective effect of thymoquinone against cyclophosphamide-induced hemorrhagic cystitis through inhibiting DNA damage and upregulation of Nrf2 expression. Int J Biol Sci. 2016;12:944. doi:10.7150/ ijbs. 15781

31. Han JH, Lee J, Jeon SJ, et al. In vitro and in vivo growth inhibition of prostate cancer by the small molecule imiquimod. Int $J$ Oncol. 2013;42:2087-2093. doi:10.3892/ijo.2013.1898

32. Mayur K, Padhi B, Chougule M, Mishra A. Methotrexate loaded solid lipid nanoparticles for topical treatment of psoriasis: formulation and clinical implication. 2002.

33. Yang G, Wu F, Chen M, Jin J, Wang R, Yuan Y. Formulation design, characterization, and in vitro and in vivo evaluation of nanostructured lipid carriers containing a bile salt for oral delivery of gypenosides. Int J Nanomed. 2019;14:2267. doi:10.2147/IJN.S194934

34. Gokhale JP, Mahajan HS, Surana SS. Quercetin loaded nanoemulsion-based gel for rheumatoid arthritis: in vivo and in vitro studies. Biomed Pharmacother. 2019;112:108622. doi:10. 1016/j.biopha.2019.108622

35. Jenning V, Thünemann AF, Gohla SH. Characterisation of a novel solid lipid nanoparticle carrier system based on binary mixtures of liquid and solid lipids. Int J Pharm. 2000;199:167-177. doi:10.1016/ S0378-5173(00)00378-1

36. Elmowafy M, Shalaby K, Ali HM, et al. Impact of nanostructured lipid carriers on dapsone delivery to the skin: in vitro and in vivo studies. Int J Pharm. 2019;572:118781. doi:10.1016/j.ijpharm. 2019.118781

37. Chen Y, Zhou L, Yuan L, et al. Formulation, characterization, and evaluation of in vitro skin permeation and in vivo pharmacodynamics of surface-charged tripterine-loaded nanostructured lipid carriers. Int J Nanomed. 2012;7:3023.

38. Agrawal Y, Petkar KC, Sawant KK. Development, evaluation and clinical studies of Acitretin loaded nanostructured lipid carriers for topical treatment of psoriasis. Int J Pharm. 2010;401(1-2):93-102. doi:10.1016/j.ijpharm.2010.09.007

39. Lee SY, Nam S, Kim S, et al. Therapeutic efficacies of Artemisia capillaris extract cream formulation in imiquimod-induced psoriasis models. Evid-Based Complement Alternat Med. 2018;2018.

40. Na Takuathung M, Wongnoppavich A, Panthong A, et al. Antipsoriatic effects of wannachawee recipe on imiquimod-induced psoriasis-like dermatitis in BALB/c mice. Evid-Based Complement Alternat Med. 2018;2018.

41. Dalle-Donne I, Milzani A, Gagliano N, Colombo R, Giustarini D, Rossi R. Molecular mechanisms and potential clinical significance of S-glutathionylation. Antioxidant Redox Signal. 2008;10:445-474. doi:10.1089/ars.2007.1716

42. Allen R, Tresini M. Oxidative stress and gene regulation. Free Radical Biol Med. 2000;28:463-499. doi:10.1016/S0891-5849(99) 00242-7

43. Zhang S, Liu X, Mei L, Wang H, Fang F. Epigallocatechin-3-gallate (EGCG) inhibits imiquimod-induced psoriasis-like inflammation of BALB/c mice. BMC Complement Alternative Med. 2016;16:334. doi:10.1186/s12906-016-1325-4 


\section{Publish your work in this journal}

The International Journal of Nanomedicine is an international, peerreviewed journal focusing on the application of nanotechnology in diagnostics, therapeutics, and drug delivery systems throughout the biomedical field. This journal is indexed on PubMed Central, MedLine, CAS, SciSearch ${ }^{\circledR}$, Current Contents ${ }^{\circledR} /$ Clinical Medicine,
Journal Citation Reports/Science Edition, EMBase, Scopus and the Elsevier Bibliographic databases. The manuscript management system is completely online and includes a very quick and fair peer-review system, which is all easy to use. Visit http://www.dovepress.com/ testimonials.php to read real quotes from published authors. 